\title{
Autonomous Fire Safety System for Gas Leak Detection
}

\author{
Jibreal Khan \\ Nizar Al-Bassam
}

\author{
Middle East College \\ Middle East College
}

\begin{abstract}
LPG and Natural Gas are the two primary gases used in Gas cookers all over the world, when these Gases leak in high concentration in enclosed areas the results can be fatal. Keeping this situation in mind this paper proposes a solution which prevents the buildup of flammable gases in enclosed area by autonomously opening windows through linear actuators. The concentration of flammable gas in a room can be detected through the MQ- 5 sensor, once the concentration of this gas exceeds the predefined threshold MQ-5 sensor sends an output to the Raspberry Pi which takes a series of steps in order to prevent the situation from taking a fatal turn. The steps include: Opening the Windows in order to prevent the buildup of flammable gas, Alerting the Residents of the house via an E-mail and an indoor alarm, Sending the precise location of the house (GPS co-ordinates) to a fire station. All these steps are done autonomously in real time, thereby responding to the situation in the most efficient way
\end{abstract}

\section{Introduction}

According to The High-Pressure Gas Safety Institute of Japan there have been 206 accidents due to the leakage of LPG in the year 2018 [1] . With the growing number of applications of LPG, the number of incidents caused due to its leakage is predicted to increase. The accumulation of LPG in contained spaces can lead to disastrous results. As LPG is a highly flammable gas it is often used in Gas cookers and other Industries as fuel. If such devices are left switched on and unmonitored for a long time the constant leakage of LPG quickly builds up and can catch fire or cause an explosion if triggered by a catalyst such as spark or high temperature. Keeping this problem in mind, the paper proposes a system which detects such leakages and immediately opens the surrounding windows in order to prevent the buildup of the flammable gas while simultaneously sending an alert Email to the user and the Fire Safety Station.

The alert email contains information such as the location of the Gas leak and the current temperature at that location. This information enables the user and the Fire safety authorities to take the best plan of action. The system requires the use of two sensors. The MQ- 5 sensor to detect gas leak and the IR flame sensor to detect fire. The MQ-5 sensor detects the presence of the Flammable gas in parts per million ( $\mathrm{ppm}$ ). When the PPM exceeds a certain pre-programmed limit, the system is activated. The IR flame sensor sends a high output to the system when it detects the light emitted by the flames. The wavelength of the light emitted by the flames are detected by the sensor and thereby the system is activated. Once the system detects the Gas leak it powers on the linear actuator to slide or push open the windows present in that room/area. A linear actuator is a device which converts rotational motion to linear motion and can be used for a variety of applications that require a push or a pull force. An alert is sent using Simple Mail Transfer Protocol (SMTP). which is implemented using a Python script. Depending upon the output of the IR flame sensor and the MQ-5 gas sensor the system sends an Alert to either the user or both the user and the fire department.

\section{Literature Review:}


Metta Santiputri and Muhammad Tio have proposed a system capable of detecting gas leaks and notifying the user via an android app. The system uses a NodeMCU , MQ-2 Gas sensor and an IR flame sensor. The type of notification received by the user depends upon the MQ sensor and the IR flame sensor. The 4 possible notifications are as follows: Android Bar notification , Alarm , Text message and beep sound produced by the system which can only be heard when the user is in proximity [2].

Cicero , Roberlanio and Marly proposed a system for detecting natural gas leaks in the oil and gas industry. The system consisted of a CCD camera and IR illumination. The system used classifiers and other methods to distinguish between images with a gas leak from images without a gas leak. The system was tested in various scenarios such as Night, Day, in the presence of rain and in the presence of humans. The system showed promising results, showing sensitivity and specificity of $94 \%$ and $96 \%$ respectively. Though such a system is extremely accurate, the system requires a camera to be fitted near the source of gas leaks [3].

Zavorotnyi and Yakimenko have proposed a Smart Sensor capable of detecting all gases which differ in their characteristics from air. Unlike other gas sensors which rely on the chemical interaction between the gas and a sensitive element, this smart sensor uses heat waves to determine the presence of gas. Thus, this Smart Sensor does not require periodic service. The sensor is built using MEMS (micro-electro-mechanical systems) structure and PSoC (programmable system on chip) [4]. 


\section{Journal of Student Research}

Fourth Middle East College Student Research Conference, Muscat, Sultanate of Oman

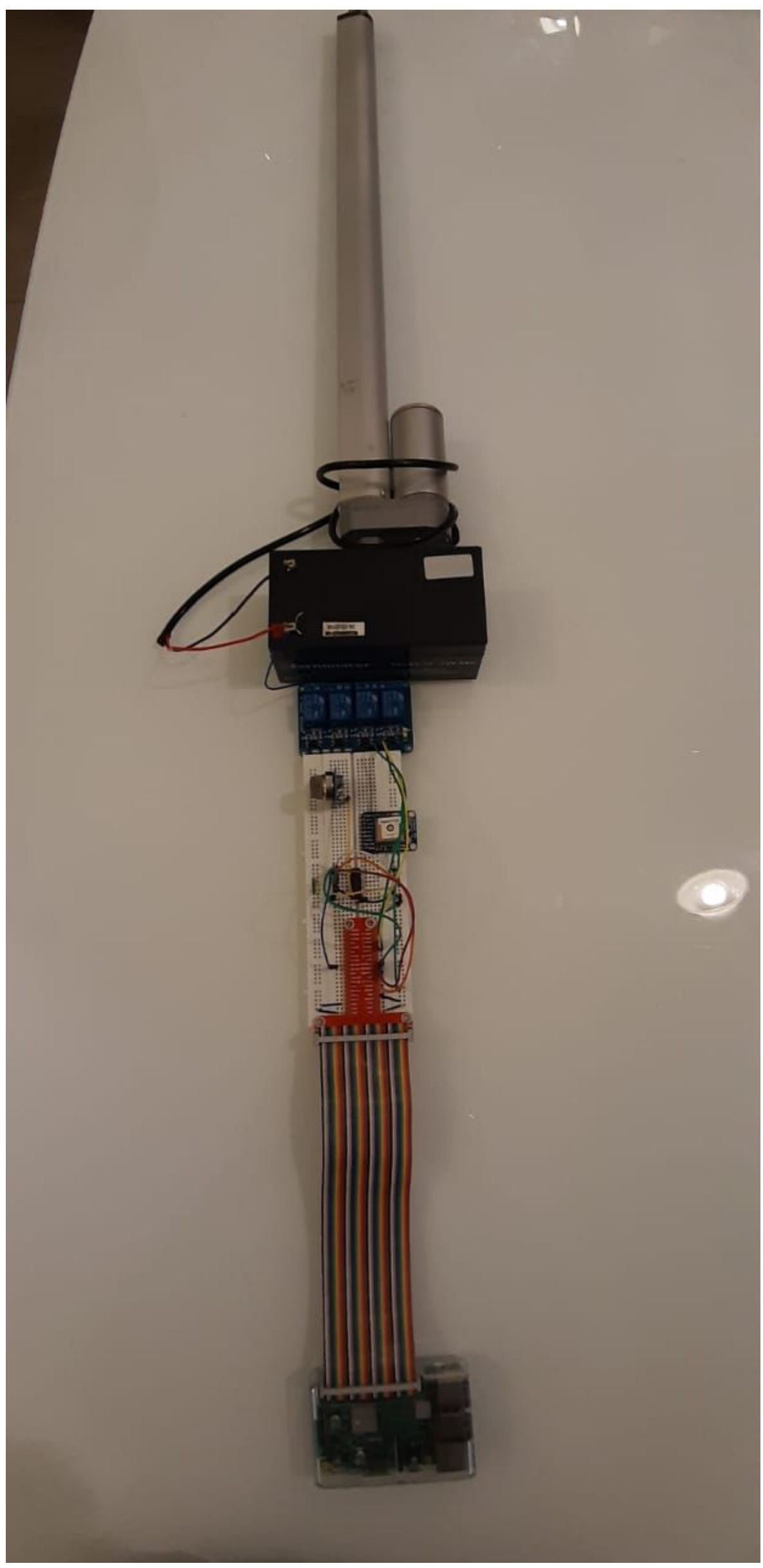


Figure 1. System Prototype

\section{Hardware description and requirements:}

The fire system consists of Raspberry Pi 3B+, 1 channel relay, 12V battery , a linear actuator , a MQ-5 gas sensor, IR Flame Sensor, DHT11 Temperature sensor, GPS module and a buzzer.

The purpose of using Raspberry Pi 3 is to make the system autonomous by controlling the output devices based on the information given by the input sensors. In this system Raspberry pi is programmed using Python language. Along with it being easily programmable, Raspberry pi 3 B consists of 28 input/output.

An electro-mechanical linear actuator is used for the purpose of sliding open windows, it converts rotational motion into linear motion and thereby is perfect to slide windows either horizontally or vertically. Raspberry pi is capable of supplying a maximum output voltage of $5 \mathrm{~V}$, as the linear actuator requires a voltage of $12 \mathrm{~V}$ an external battery is used along with a 1-channely relay. The stroke length of the linear actuator is $400 \mathrm{~mm}$ and the stroke speed is about $60 \mathrm{~mm} / \mathrm{s}$ when no load is being pushed by it.

The MQ-5 sensor is suitable for detecting LPG , Natural gas, Town gas, etc. LPG is the gas most commonly used for cooking and MQ-5 sensor has high sensitivity to LPG. The MQ-5 gas sensor consists of a sensitive filament made of $\mathrm{SnO} 2$. The resistance of this filament drops when it comes in contact with a combustible gas such as LPG. This change is resistance of the filament is used to indicate the presence of LPG in the air.

The IR Flame sensor is used to detect the presence of flames. The flame sensor used in this system is sensitive to Infrared Radiation in the range of $760 \mathrm{~nm}$ to $1100 \mathrm{~nm}$. Typically, the light emitted by flames lies in this range and is thereby detected by the flame sensor.

The DHT11 sensor is used to detect the temperature of the environment. The surrounding temperature can be an indicator of the presence of fire. The DHT11 sensor consists of a Negative Temperature Coefficient (NTC) element whose resistance changes with change in temperature. This change in resistance is used to determine the surrounding temperature.

The Adafruit-Gps module is used for the purpose of extracting the longitude and latitude of the location. The GPS is capable of tracking up to 22 satellites. The power consumed by the module is as low as $20 \mathrm{~mA}$ during navigation and it is capable of receiving 10 location updates per second. The module has an inbuilt antenna which at times is unable to lock on to the satellites. However, an external antenna can be attached to the GPS module using a uFL to SMA adapter. 


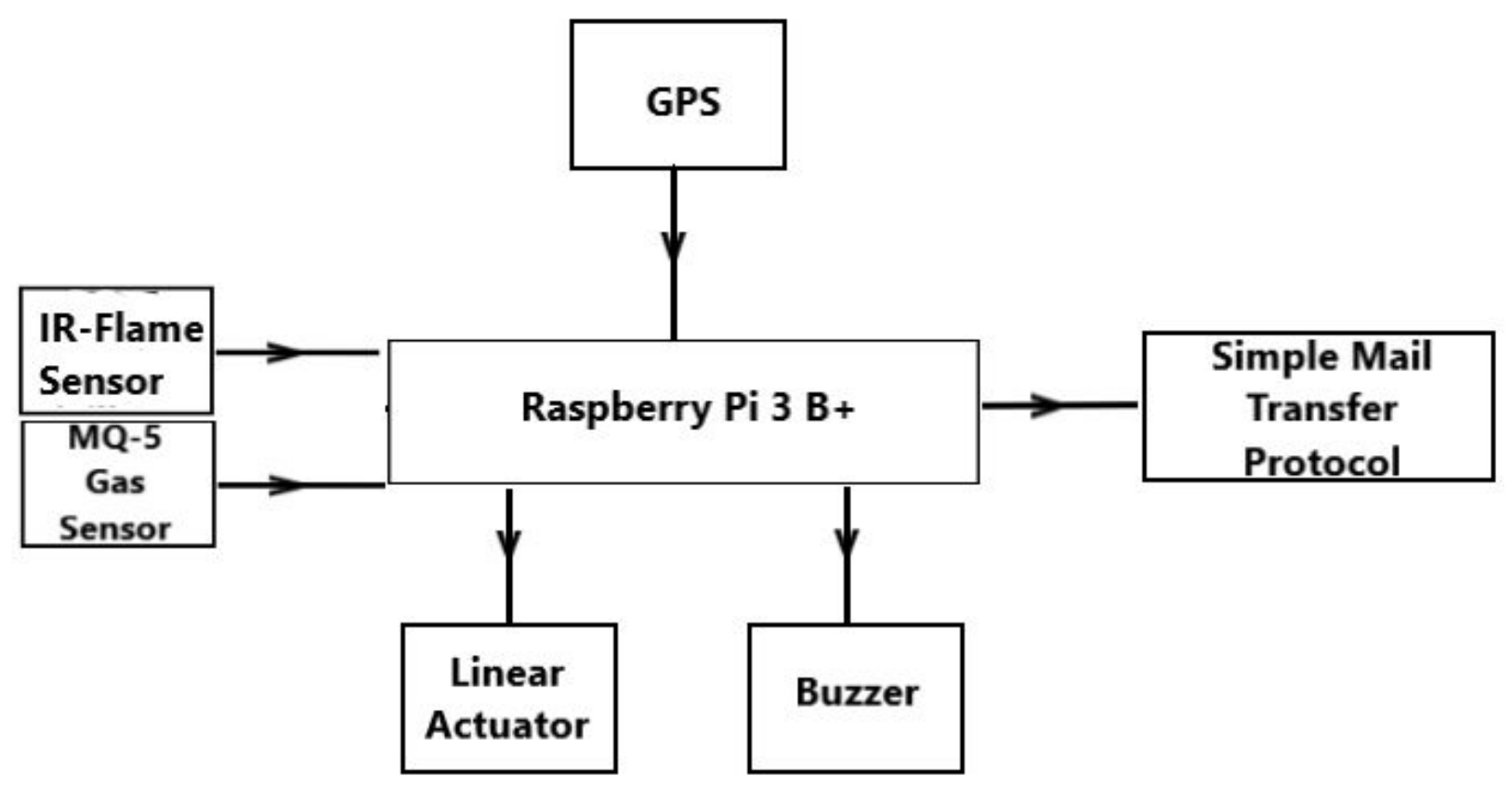

Figure 2. System Block Diagram

\section{System Analysis}

Once the LPG or Natural gas is detected by the sensor, it sends an output to the Raspberry pi through the digital out pin. When the output received matches the programmed conditions , a series of steps are taken using the "If Else " operators. The raspberry turns on the linear actuator by activating the relay to which the external battery and the linear actuator are connected. This switches on the linear actuator and opens the window present in the environment in order to prevent the flammable gas from building up in a closed area. The co-ordinates of the location where the system is located are extracted using the Adafruit GPS module. The process of locking on to satellites can take as long as 2 mins, once the GPS locks on it starts receiving information from the satellites in the form of NMEA sentences. The data obtained from the GPS module is raw data and consists of additional unrequired information. Only the Latitude and Longitude are extracted from the raw data. and sent to the user using SMTP (Simple Mail Transfer Protocol). The email address of the user/user's is preprogrammed in the system. SMTP is achieved using a set of pre-compiled libraries in Python 3. 
Figure 3. System Flowchart

\section{Pseudo Code:}

If $($ Gas is detected $=$ True $)$

\{

Linear Actuator $=$ High

Buzzer pin $=$ High

Function (Get Co-ordinates using GPS):

\{

(Latitude, Longitude) $=$ NMEA sentences

\}

Function (Sending a Email to the user):

\{

Email content $=($ Latitude, Longitude $)$

Send (Email content)

\} .

If (Flame is detected $=$ True $)$

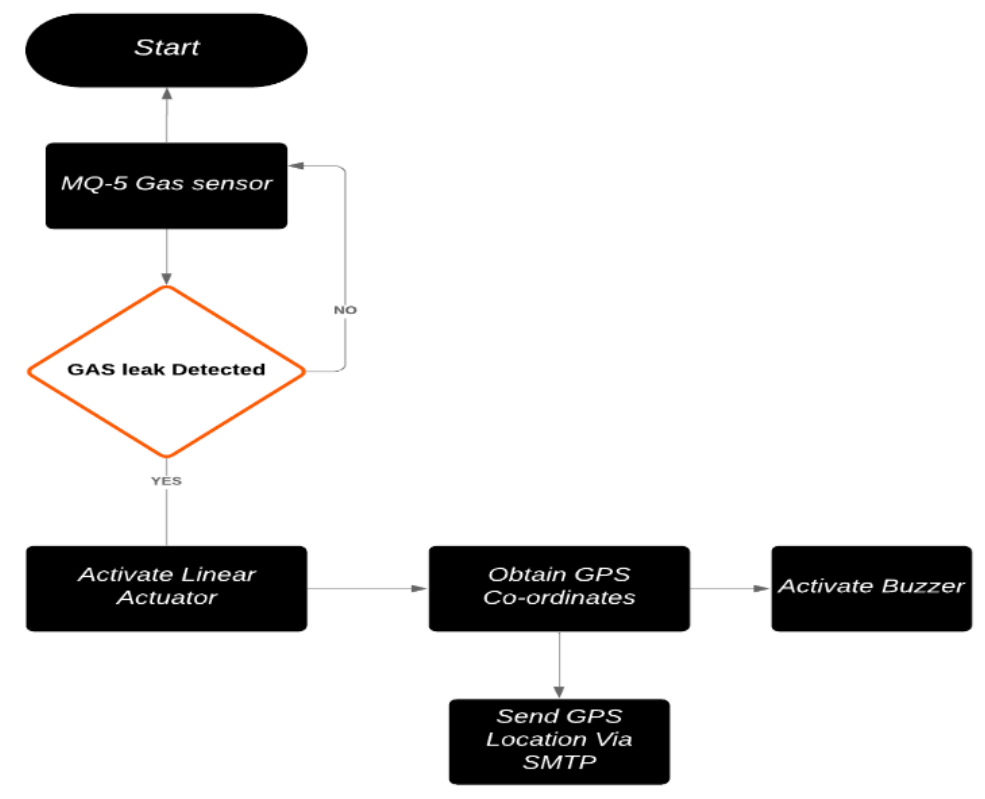


Function (Get Co-ordinates using GPS):

\{

$($ Latitude, Longitude $)=$ NMEA sentences

\}

Function (Sending a Email to the Fire Station and user):

\{

Email content $=($ Latitude, Longitude $)$

Send (Email content)

\} .

\}

\section{System Testing}

\section{Testing the MQ-5 sensor}

The MQ-5 gas sensor yields output in terms of Parts per million which describes the concentration of the gas. In order for an explosion to occur the combustible gas should be present in certain proportion with respect to Oxygen. This ratio of Oxygen and fuel varies from one combustible gas to other. The minimum required concentration for a particular gas to burn is called Lower explosive limit (LEL). The maximum concentration at which the gas can burn in air is called Upper explosive limit (UEL) . Above the UEL the mixture become too rich to burn. Therefore, the flammable range of a gas lies between LEL and UEL. Since LPG has a LEL of $1.8 \%$ and an UEL of $9.8 \%$, the system must alert the user before the concentration of LPG reaches the flammable range.

Figure 4. $M Q-5$ sensor calibration

\section{Testing the Speed of Sliding the Window:}

Sparks are often the catalyst for explosions caused due to Gas leaks. The system uses an external $12 \mathrm{v}$ battery to power the linear actuator which could cause a spark. As an added measure of safety, the Linear actuator should operate only when the concentration of gas is much below the lower explosive limit. This is achieved by activating the linear actuator as soon as the gas leak is detected to prevent the concentration of the flammable gas from reaching the flammable range. Also, the stroke speed of the linear actuator should be fast enough to open the window before the buildup of gas happens.

Keeping these safety parameters in mind the system uses an actuator with a stroke speed of $60 \mathrm{~mm} / \mathrm{s}$. For experimental purposes a $60 \mathrm{~cm} \mathrm{X} 40 \mathrm{~cm}$ window frame was used.

\begin{tabular}{|l|l|}
\hline Theoretical time of the window sliding: & Experimental time of sliding the window: \\
\hline $600 / 60=10 \mathrm{~s}$ & $12 \mathrm{~s}$ \\
\hline & \\
\hline
\end{tabular}


Table 1. Sliding using Linear Actuator

The difference between the values is due to the friction offered by the window frame.

\section{Extracting Location for NMEA sentences:}

The Adafruit GPS module provides data to the Raspberry pi in the form of NMEA sentences. These NMEA sentences contain data such as Latitude, Longitude, Number of Satellites being Tracked, Horizontal dilution of position, Altitude, DGPS station ID number etc. [5] The data is provided in a complicated format that is unreadable to an average person.

\$GPRMC,123519,A,4807.038,N,01131.000,E,022.4,084.4,230394,003.1,W*6A

In order to make this data readable a Python script is used to extract only the necessary data from the NMEA sentence and arrange them in a user-friendly format.

Figure 5. Data Extraction for NMEA sentences

The co-ordinates are then passed as Query parameters to the Google maps URL which is later sent to the user and the fire safety authorities via email.

Figure 6. Location Sent using Google Maps via Email

\section{GPS satellite lock on time:}

The Adafruit GPS breakout comes with an internal antenna. The GPS module takes around 1-3 mins to lock on to satellites and provide an accurate location. However, at times the module is unable to lock on to any satellites due to the environment it is placed in. In such situations, an external Antenna is more suitable.

\section{Alert using SMTP (Simple Mail transfer protocol)}

Upon the detection of gas leak the system alerts the user and the authorities by sending them an email. This action is performed using the SMTP library for Python. Details such as the receivers ID and the sender's login credentials are coded in the circuit. The server used to provide this functionality is Gmail. The email consists of data such as the Location of the place where the gas leak was detected, the type of Gas that has been leaked and weather the gas has caught fire or not.

The are 2 conditions in which an email is sent, the first condition is that the MQ-5 sensor detects the leakage of a Flammable gas and no fire is detected by the IR flame sensor. In this case, an email is sent only to the user and not the fire department. The second condition is that the IR sensor detects the presence of flames. In this case , an email is sent to the Fire department along with the user.

\begin{tabular}{|l|l|l|}
\hline Output of the Flame sensor & Output of the Gas sensor & Output of the System \\
\hline Low & High & Email sent to the User \\
\hline High & Low & Email sent to the Fire dept and user \\
\hline High & High & Email sent to the Fire dept and user \\
\hline Low & Low & No Alert Sent \\
\hline
\end{tabular}

Table 2. Conditions for Alerting 


\section{Conclusion and Recommendation:}

The system was designed in order to prevent LPG gas from being contained in closed areas which could lead to a fire outbreak. The system detects LPG with the help of MQ-5 sensor which signals the Raspberry pi about the presence of LPG. The Raspberry pi performs a series of tasks such as opening the windows using linear actuators, sending an alert mail to the user or the fire department. Where the email contains helpful information such as the location where the gas leak has been detected. Such a system that works completely autonomously can potentially save lives if implemented on a large scale. A few features that can be added on to the system are : attachment of a GSM module to provide a different way of alerting the user, implementation of haversine law such that an email sent to the closest fire station from the place of incident and addition of an electronic valve that could shut of the supply of gas from the cylinder upon the detection of gas leakage.

\section{Acknowledgement:}

The research and experiments were conducted under the facilities provided by the Department of Electronics and Telecommunication in Middle East College.

\section{References:}

1. Khk.or.jp. (2018). Annual Report on Liquefied Petroleum Gas Related Accidents. [online] Available at: https://www.khk.or.jp/Portals/0/khk/info/2018/LPG\%20related\%20accidents\%202018.pdf [Accessed 22 Nov. 2019].

2. Santiputri, M. and Tio, M. (2018). IoT-based Gas Leak Detection Device. 2018 International Conference on Applied Engineering (ICAE). [online] Available at: https://ieeexplore.ieee.org/document/8579396 [Accessed 22 Nov. 2019].

3. Filho, Cicero \& Melo, Roberlânio \& Costa, Marly. (2013). An improved system for detecting natural gas leaks. Proceedings of 2013 Science and Information Conference, SAI 2013. 443-447.

4. Zavorotnyi, V. \& Yakimenko, Yu. (2009). Based on PSoC Smart Sensor of gas leakage. 10.1109/ISSE.2009.5206973.

5. DePriest, D. (2019). NMEA data. [online] Gpsinformation.org. Available at: https://www.gpsinformation.org/dale/nmea.htm [Accessed 23 Nov. 2019]. 